\title{
近似贝叶斯法在光合模型参数估计中的应用
}

\author{
曾继业 ${ }^{1}$ 谭正洪 ${ }^{2 *}$ 三枝信子 ${ }^{1}$ \\ ${ }^{1}$ 日本国立环境研究所, 筑波 305-8506, 日本; ${ }^{2}$ 海南大学环境科学系, 海口 570228, 中国
}

摘 要 长期以来, 光合作用机理模型中参数的确定都是一个难点。该文提出一种参数反演的方法, 称为近似贝叶斯法 (APMC), 用来确定Farquhar光合模型的生理参数。通过将整个冠层抽象为一片大叶的思维抽象, 笔者进一步将APMC应用到 冠层尺度的生理参数求解, 使直接求算冠层尺度生理参数成为可能。该文详细介绍了使用APMC估算光合模型参数的具体算 法, 并用实测数据进行了验证。结果表明, APMC可以很好地应用于冠层光合模型参数的估计, 估计所得的参数落在参数生理 上下限值之间, 应用 1948 个实测数据进行检验, 得到决定系数 0.75 。模拟值和实测值的线性回归曲线斜率为 1.04 , 与理论上的 1.0 非常接近。这个方法对光合模型参数的获取或许有积极的意义。

关键词 蒙特卡洛; 大叶模型; Farquhar光合模型; 净生态系统交换

引用格式: 曾继业, 谭正洪, 三枝信子 (2017). 近似贝叶斯法在光合模型参数估计中的应用. 植物生态学报, 41, 378-385. doi: 10.17521/cjpe.2016.0067

\section{Using approximate Bayesian computation to infer photosynthesis model parameters}

ZENG Ji-Ye ${ }^{1}$, TAN Zheng-Hong ${ }^{2 *}$, and SAIGUSA Nobuko ${ }^{1}$

${ }^{1}$ National Institute for Environmental Studies, Tsukuba 305-8506, Japan; and ${ }^{2}$ Department of Environmental Science, Hainan University, Haikou 570228, China

\begin{abstract}
We developed a method, namely Adaptive Population Monte Carlo Approximate Bayesian Computation (APMC), to estimate the parameters of Farquhar photosynthesis model. Treating the canopy as a big leaf, we applied this method to derive the parameters at canopy scale. Validations against observational data showed that parameters estimated based on the APMC optimization are un-biased for predicting the photosynthesis rate. We conclude that APMC has greater advantages in estimating the model parameters than those of the conventional nonlinear regression models.
\end{abstract}

Key words Monte Carlo; big-leaf model; Farquhar photosynthesis model; net ecosystem exchange

Citation: Zeng JY, Tan ZH, Saigusa N (2017). Using approximate Bayesian computation to infer photosynthesis model parameters. Chinese Journal of Plant Ecology, 41, 378-385. doi: 10.17521/cjpe.2016.0067

光合作用利用光能进行水的光解, 释放 $\mathrm{O}_{2}$, 同 化大气中的 $\mathrm{CO}_{2}$, 形成有机物, 是生态系统生产力 形成的基础, 也是能量流动和物质循环的起点。准 确模拟光合作用具有重要的科学意义和应用价值。 在20世纪80年代初期，随着Farquhar光合作用生化 过程机理模型的提出, 这项工作有了长足的进展 (Farquhar et al., 1980)。现今, Farquhar光合模型有了 非常广泛的应用, 已成为叶片尺度光合作用模拟的 主流机理模型。驱动模型运行的一些气象因子, 比 如辐射、温度和湿度等, 在具体应用中容易获得。 模型应用的一个难点是生理参数的确定, 特别是最 大翔化速率 $\left(V_{\mathrm{cmax}}\right)$ 和最大电子传输速率 $\left(J_{\max }\right)$ 的确 定。众所周知, 生理参数 $V_{\mathrm{c} \text { max }} 、 J_{\mathrm{max}}$ 在不同物种间差
异很大, 同一个物种在不同季节间的变化也很显著 (Xu \& Baldocchi, 2003), 想要获得这些参数并将其 应用到区域或者全球尺度的研究中, 难度很大。模 型应用的另一难点在于尺度的扩展, Farquhar光合 模型是叶片尺度的模型, 若想将该模型运用到冠层 或者更大尺度的研究中, 需要进行合理的尺度转换 (Jarvis, 1995), 而这种尺度转换是生态学研究中尚 未解决的问题(Amthor, 1994)。以热带森林为例, 测 定数以百计的树种的 $V_{\mathrm{cmax}}$ 和 $J_{\max }$, 即使不考虑人力 物力上的巨大投入, 如何将测定结果组织起来, 以 便可以代表整个冠层, 目前还未找到合适的方案 (Sprintsin et al., 2012; Cubasch et al., 2013)。因此, 亟 需寻找到一种合理的尺度扩展方案, 作为独立的、 
可靠的验证标准, 冠层尺度的 $V_{\mathrm{cmax}}$ 和 $J_{\max }$ 直接实测 值是必不可少的。

浴度相关技术(eddy covariance technique)作为 目前直接测定地表-大气间 $\mathrm{CO}_{2}$ 和水热通量的标准 方法, 为原位、无破坏监测生态系统的光合作用(实 质上植被大气之间的 $\mathrm{CO}_{2}$ 交换)提供了新的选择(de Pury \& Farquhar, 1997; Wang \& Leuning, 1998; Dai et al., 2004; Groenendijk et al., 2011; Kosugi et al., 2013)。在过去的30余年里, 该方法有了快速的发展, 得到了广泛的应用, 目前已成为国际通量观测网络 (FLUXNET)的主要技术手段。据不完全统计, 目前 全球安装涡度相关监测系统的研究站已经超过 5000 个, 仅中国就超过了 300 个。考虑到浴度相关法 可以提供冠层光合作用的实测值, 而叶片尺度的光 合模型也很明确, 笔者认为可以在叶片光合模型的 基础上提出一种算法, 直接反演出 $V_{\mathrm{cmax}}$ 和 $J_{\max }$ 这两 个重要的生理参数在冠层上的数值。如果这一想法 能够实现, 我们就有可能从生理学角度切入, 对大 量冠层尺度的监测数据进行更深入的解读和挖掘。 作为一个初步的尝试, 为了简化模型的数据和计算, 本文通过将冠层抽象为一片大叶, 提出了一种相对 简洁却行之有效的方法来反演冠层的生理参数。下 文中, 笔者将对该方法的技术细节进行分解, 希望 该方法能引起读者的兴趣, 在读者的批评中获得进 一步提高和完善。

\section{1 方法}

\section{1 贝叶斯计算法}

统计分析经常面对的一个挑战是用观测数据 估计多元非线性模型的参数。

$$
y=f(x, \theta)+\varepsilon
$$

在上式中, $x$ 为自变量, $\theta$ 为待定参数向量, $\varepsilon$ 是模拟对 象 $y$ 变量的观测误差。基于频率统计学(frequentist statistics)的参数估计方法, 一般是在假定 $\varepsilon$ 服从某一 统计分布的条件下, 通过优化求出固定的 $\theta$ 值, 该值 的可信度, 或在某一可信度下包含 $\theta$ 真值的范围。而 基于贝叶斯统计学(Bayesian statistics)的方法, 则是 假定 $\theta$ 服从某一先验分布 $\pi(\theta)$ (prior distribution), 依 据贝叶斯概率法则, 通过优化估计 $\theta$ 的后验分布 (posterior distribution):

$$
p(\theta \mid y)=\frac{p(y \mid \theta) \pi(\theta)}{p(y)}
$$

$$
p(y)=\int p(y \mid \theta) \pi(\theta) \mathrm{d} \theta
$$

其中, $p(y \mid \theta)$ 为似然函数(likelihood function), $p(y)$ 为边 缘似然函数(marginal likelihood function)。当似然函 数和边缘似然函数易得, 或可以通过某种近似法, 诸如马尔可夫链蒙特卡洛法(Markov chain Monte Carlo, Andrieu et al., 2010)、重点取样法(importance sampling, MacEachern et al., 1999)以及序贯蒙特卡 洛法(sequential Monte Carlo, Gao \& Zhang, 2012)等 算出时, 这些方法便可用来求解待定参数的后验分 布。然而对复杂的非线性多参数模型而言, 似然函 数往往不可知, 或者计算边缘似然函数有困难。在 此背景下, 近似贝叶斯计算法(Approximate Bayesian Computation)应运而生(Toni et al., 2009; Beaumont, 2010; Marin et al., 2012), 并被广泛应用 (Csilléry et al., 2010; Vrugt \& Sadegh, 2013; Hartig et $a l ., 2014)$ 。在此, 我们介绍如何应用Lenormand等 (2013) 提出的自调近似贝叶斯计算法(APMC)来估 计植物光合模型的几个重要参数。

\section{2 光合作用模型}

基于将冠层视为一片大叶的思维抽象, 叶片尺 度的光合模型便得以直接应用于冠层之上, 简称大 叶模型。本文采用了Farquhar模型来模拟冠层尺度 的光合作用, 同时采用Ball-Berry模型来模拟气孔 导度关系(Ball et al., 1987)。具体使用到的关系式见 表1; 各缩写和符号代表的含义和参数初值见表2。 模型细节请参考Medlyn等(2002a), von Caemmerer 等(2009)和Damour等(2010)的文献。

\subsection{APMC算法的具体运行}

首先我们假设大叶模型的叶面 $\mathrm{CO}_{2}$ 分压 $\left(C_{\mathrm{s}}\right)$ 、 胞内氧分压 $\left(O_{\mathrm{i}}\right)$ 、叶面相对湿度 $\left(h_{\mathrm{s}}\right)$ 可以用环境空气 的实测值近似代替(附录I)。之所以做出如此假设, 是因为: 一方面, 我们无法知道哪些叶片的 $C_{\mathrm{s}} 、 O_{\mathrm{i}}$ 、 $h_{\mathrm{s}}$ 值能代表整个冠层; 另一方面, 从大多数过程模 型使用的边界层导度模块来看, 这些参数值在叶片 表层与大气之间相差不会太大。但是, 胞间 $\mathrm{CO}_{2}$ 浓度 $\left(C_{\mathrm{i}}\right)$ 是个例外，它是整个模型运行的一个关键，不 能直接假定其与环境 $\mathrm{CO}_{2}$ 浓度相等。用来估计 $C_{\mathrm{i}}$ 的 方法主要有两种，一种方法是通过迭代得到，另一 种方法是用Baldocchi (1994)提出的3次方程解。虽然 Baldocchi (1994)、Yin和Struik (2009)等都认为3次方 程解适用于正向模拟的绝大多数情况, 但我们的实 
践表明它不适用于逆向参数估计, 因为逆向模拟时, 参数值变化范围可能很大, 因此本文最终采用了迭 代法。我们在实践中还发现, 大多数情况下只需要 低于10次的迭代就可得到稳态的 $C_{\mathrm{i}}$ 解。

表1中的方程(10)是控制光合速率的关键方程。 它实际上是在Arrhenius方程(9)基础上引入了

$$
f(S, H)=\frac{1+\mathrm{e}^{(298 S-H) /(298 R)}}{1+\mathrm{e}^{(T K \times S-H) /(T K \times R)}}
$$

进行温度修正, 使得 $V_{\mathrm{cmax}} 、 J_{\max }$ 在高温下减小。正如 图1所示, 修正曲线对某些范围内的参数 $S$ 和 $H$ 的变 化很敏感。作为实例, 我们仅把von Caemmerer等 (2009)温度修正模型中的 $S$ 值增加约 $10 \%$, 就使修正 曲线系数的变化区间由 $(0.0,1.1)$ 扩大到 $(0.0,420)$, 并在 $0{ }^{\circ} \mathrm{C}$ 附近开始急剧下降。这种敏感性对于正向 模拟不构成问题, 因为参数调试时可以根据经验, 不断缩小范围逐步进行调整。而在逆向模拟参数反 演时, 参数调试依据的是规则而非经验, 这种敏感 性会导致非优化解。针对这一问题, Medlyn等(2002b) 的处理方法是固定 $H$ 的值, 通过详细的分析和探索, 我们建立了一个温度修正方程, 可以避免参数反演 时数值不稳定性, 同时得到优化的反演参数。这个 新的温度修正曲线表达如下:

表1 光合作用机理模型的基本方程(含气孔模型)

Table 1 Algorithms for photosynthesis biochemical model at canopy level

\begin{tabular}{|c|c|}
\hline 序号 Number & 方程 Equation \\
\hline 1 & $A_{\mathrm{c}}=\frac{\left(C_{\mathrm{i}}-\Gamma^{*}\right) V_{\mathrm{cmax}}}{C_{\mathrm{i}}+K_{\mathrm{c}}\left(1+O_{\mathrm{i}} / K_{\mathrm{o}}\right)}-R_{\mathrm{d}}$ \\
\hline 2 & $A_{\mathrm{j}}=\frac{\left(C_{\mathrm{i}}-\Gamma^{*}\right) J}{4 C_{\mathrm{i}}+8 \Gamma^{*}}-R_{\mathrm{d}}$ \\
\hline 3 & $0.98 A^{2}-\left(A_{\mathrm{c}}+A_{\mathrm{j}}\right) A+A_{c} A_{\mathrm{j}}=0$ \\
\hline 4 & $C_{\mathrm{i}}=C_{\mathrm{a}}-A\left(\frac{1}{g_{\mathrm{b}}}+\frac{1}{g_{\mathrm{s}}}\right)$ \\
\hline 5 & $g_{\mathrm{b}}=0.147 \times \sqrt{\frac{W S}{0.72 \times \text { LeafW }}}$ \\
\hline 6 & $g_{\mathrm{s}}=\frac{g_{1} h_{\mathrm{s}}}{C_{\mathrm{s}}} A+g_{0}$ \\
\hline 7 & $I_{2}=I_{0} \times(1-f) \times(1-\alpha) / 2$ \\
\hline 8 & $0.7 J^{2}-\left(I_{2}+J_{\text {max }}\right) J+I_{2} J_{\max }=0$ \\
\hline 9 & $\left\{K_{\mathrm{c}}, K_{\mathrm{o}}, R_{\mathrm{d}}, \Gamma^{*}\right\}=\left\{K_{\mathrm{c} 25}, K_{\mathrm{o} 25}, R_{\mathrm{d} 25}, \Gamma_{25}^{*}\right\} \mathrm{e}^{E_{\mathrm{s}} \frac{T K-298}{298 \times R \times T K}}$ \\
\hline 10 & $\left\{V_{\text {cmax }}, J_{\max }\right\}=\left\{V_{25}, J_{25}\right\} \mathrm{e}^{E_{s} \frac{T K-298}{298 \times R \times T K}} \frac{1+\mathrm{e}^{(298 S-H)(298 R)}}{1+\mathrm{e}^{(T K \times S-H)(T K \times R)}}$ \\
\hline 11 & $\left\{V_{\text {cmax }}, J_{\max }\right\}=\left\{V_{\text {opt }}, J_{\text {opt }}\right\} \mathrm{e}^{E_{\frac{1}{2}} \frac{T K-298}{298 \times R \times T K}} \frac{1+\mathrm{e}^{-C_{\mathrm{m}} T_{\mathrm{m}}}}{1+\mathrm{e}_{\mathrm{m}}^{C_{\mathrm{m}} \times\left(T C-T_{\mathrm{m}}\right)}}$ \\
\hline
\end{tabular}

缩写同表2。

Abbreviations see Table 2.

$$
f\left\{C_{\mathrm{m}}, T_{\mathrm{m}}\right\}=\frac{1+\mathrm{e}^{-C_{\mathrm{m}} T_{\mathrm{m}}}}{1+\mathrm{e}^{C_{\mathrm{m}} \times\left(T C-T_{\mathrm{m}}\right)}}
$$

事实上, Seller等(1996)也用了类似的方程来修 正Rubisco的活化能。新的温度修正曲线与June等 (2004)提出的简易温度修正的不同之处是，新修正曲 线可以很好地拟合 $f(S, H)$, 且修正系数的变化范围 小, 避免了一些数值计算问题(图1)。并且, 我们可 以比较直观地选择 $C_{\mathrm{m}}$ 和 $T_{\mathrm{m}}$ 的可变范围。实际上, 对任 意正的 $C_{\mathrm{m}} 、 T_{\mathrm{m}}$ 和 $T C, f\left(C_{\mathrm{m}}, T_{\mathrm{m}}\right)$ 的变化只限于 $(0,1)$ 区间。

APMC算法的具体实现参见附录 $\mathrm{I}$ 。必要的输入 数据包括 $N E E 、 P P F D 、 T K 、 R H 、 W S 、 P 、 L e a f W$ 、 $C_{\mathrm{a}}$ 。选定了模型、待定参数、输入变量, 就确定了 APMC循环计算中 $y=f(x, \theta)$ 的计算。

\section{4 验证数据的获取}

验证用的数据获取自日本的苫小牧观测站 $\left(42.73^{\circ} \mathrm{N}, 141.52^{\circ} \mathrm{E}\right)$, 由AsiaFlux提供。其中净生态 系统 $\mathrm{CO}_{2}$ 交换 $(N E E)$ 白天的数值可以视为生态系统 昼间的净光合速率。 $N E E$ 是浴度碳通量和碳储量通 量之和。浴度碳通量是三维超声风速仪所测得的垂 直风速和红外气体分析仪测定的高频 $\mathrm{CO}_{2}$ 浓度值, 是在时间尺度上求协方差得到的。而储量通量则是 通过 $\mathrm{CO}_{2}$ 浓度的垂直廓线在时间的变化计算得到。 有关的观测方法、周边环境和数据处理细节等, 在 Hirano等(2003)、Wang等(2004)和 Saigusa等(2008) 的文中已有说明, 此处不再赘述。研究选择了 2003 年6月至9月期间的数据。因为此这期间, 归一化植 被指数 $(N D V I)$ 显示树叶的总量变化比较小, 使我 们可以不考虑叶面积指数 $(L A I)$ 或树叶的年龄因子 的影响。把有缺损和有效光量子密度 $(P P F D)$ 小于 100 的数据点消除后, 一共得到 1948 个完整的、 $30 \min$ 时间间隔的有效数据点。

\section{2 结果}

我们用了一万个蒙特卡洛粒子做模拟实验, 并设 $N_{a}=100$, 即每次循环计算都接受 10000 粒子 中最好的 100 个。从验证方法的角度出发, 我们不 打算讨论这 100 粒子的参数值的统计分布, 而只 比较由最佳粒子的参数值产生的 $N E E$ 与观测值之 间的差距。

拟合得到的方程参数如表 3 所示。用APMC方法 估计的参数值全部落在参数值的上下限范围内, 这 表明从植物生理学含义的角度看, 这个模拟结果是 
表2 模型的变量、参数及其参考值(主要参考Caemmerer等(2009))

Table 2 Variables and parameters used in the photosynthesis model and their reference values mainly from Caemmerer et al. (2009)

\begin{tabular}{|c|c|c|c|}
\hline 符号 Symbol & 单位 Units & 注释 Remark & 参考值 Reference value \\
\hline$A$ & $\mu \mathrm{mol} \cdot \mathrm{m}^{-2} \cdot \mathrm{s}^{-1}$ & 净光合速率 Net photosynthesis rate & \\
\hline$A_{\mathrm{c}}$ & $\mu \mathrm{mol} \cdot \mathrm{m}^{-2} \cdot \mathrm{s}^{-1}$ & Rubisco酶限制下的光合速率 Rubisco activity limited net photosynthesis rate & \\
\hline$A_{\mathrm{j}}$ & $\mu \mathrm{mol} \cdot \mathrm{m}^{-2} \cdot \mathrm{s}^{-1}$ & RuBP再生限制的光合速率 Electron transport limited net photosynthesis rate & \\
\hline$R_{\mathrm{d}}$ & $\mu \mathrm{mol} \cdot \mathrm{m}^{-2} \cdot \mathrm{s}^{-1}$ & 呼吸速率 Respiration rate & \\
\hline$C_{\mathrm{i}}$ & $\mu \mathrm{bar}$ & 胞内 $\mathrm{CO}_{2}$ 分压 Intercellular $\mathrm{CO}_{2}$ partial pressure & \\
\hline$C_{\mathrm{a}}$ & $\mu \mathrm{bar}$ & 大气 $\mathrm{CO}_{2}$ 分压 $\mathrm{Air} \mathrm{CO}_{2}$ partial pressure & \\
\hline$C_{\mathrm{s}}$ & $\mu \mathrm{bar}$ & 叶面 $\mathrm{CO}_{2}$ 分压 Leaf-surface $\mathrm{CO}_{2}$ partial pressure & \\
\hline$\Gamma^{*}$ & $\mu \mathrm{bar}$ & $\mathrm{CO}_{2}$ 补偿点 $\mathrm{CO}_{2}$ compensation point & \\
\hline$V_{\text {cmax }}$ & $\mu \mathrm{mol} \cdot \mathrm{m}^{-2} \cdot \mathrm{s}^{-1}$ & 最大羧化速率 Maximal rubisco carboxylase rate & \\
\hline$J_{\max }$ & $\mu \mathrm{mol} \cdot \mathrm{m}^{-2} \cdot \mathrm{s}^{-1}$ & 最大电子传输速率 Maximal electronic transport rate & \\
\hline$J$ & $\mu \mathrm{mol} \cdot \mathrm{m}^{-2} \cdot \mathrm{s}^{-1}$ & 电子传输速率 Electronic transport rate & \\
\hline$I_{2}$ & $\mu \mathrm{mol} \cdot \mathrm{m}^{-2} \cdot \mathrm{s}^{-1}$ & 电子传输速率 Electronic transport rate through photosystem II & \\
\hline$I_{0}$ & $\mu \mathrm{mol} \cdot \mathrm{m}^{-2} \cdot \mathrm{s}^{-1}$ & 光照强度 Photon flux density & \\
\hline$f$ & & 光谱校正常数 Fraction of effective photon flux & 0.15 \\
\hline$\alpha$ & & 转化率 Conversion efficiency & 0.15 \\
\hline$O_{\mathrm{i}}$ & mbar & 胞内氧分压 Intercellular $\mathrm{O}_{2}$ partial pressure & 210 \\
\hline$K_{\mathrm{o}}$ & mbar & 氧化酶的动力学常数 Michaelis-Menten constant of Rubisco for $\mathrm{O}_{2}$ & \\
\hline$K_{\mathrm{c}}$ & $\mu \mathrm{bar}$ & 羧化酶的动力学常数 Michaelis-Menten constant of Rubisco for $\mathrm{CO}_{2}$ & \\
\hline$g_{\mathrm{b}}$ & $\mathrm{mol} \cdot \mathrm{m}^{-2} \cdot \mathrm{s}^{-1}$ & 边界层导度 Boundary-layer conductance & \\
\hline$g_{\mathrm{s}}$ & $\mathrm{mol} \cdot \mathrm{m}^{-2} \cdot \mathrm{s}^{-1}$ & 气孔导度 Stomatal conductance & \\
\hline$g_{0}$ & $\mathrm{~mol} \cdot \mathrm{m}^{-2} \cdot \mathrm{s}^{-1}$ & 气孔最小导度 Minimum stomatal conductance & 0.01 \\
\hline$g_{1}$ & & 气孔导度常数 Sensitivity coefficient of stomatal conductance & 10.0 \\
\hline$h_{\mathrm{s}}$ & & 叶面相对湿度 Relative humidity on leaf surface & \\
\hline$W S$ & $\mathrm{~m} \cdot \mathrm{s}^{-1}$ & 风速 Wind speed & \\
\hline LeafW & $\mathrm{m}$ & 叶片宽度 Leaf width & \\
\hline $\begin{array}{l}V_{25}, J_{25}, K_{\mathrm{c} 25,} \\
K_{\mathrm{o} 25}, R_{\mathrm{d} 25}, \Gamma_{25}^{*}\end{array}$ & & 相应参数在 $25{ }^{\circ} \mathrm{C}$ 的值 Values at $25^{\circ} \mathrm{C}$ & $\begin{array}{l}\text { 分别为 } 80,160,260,165,1,38 \\
\text { respectively }\end{array}$ \\
\hline$E_{\mathrm{a}}$ & $\mathrm{J} \cdot \mathrm{mol}^{-1}$ & 活化能 Activation energy & $\begin{array}{l}\text { 分别为 } 58500,37000,59400 \text {, } \\
36000,66400,23400 \text { respectively }\end{array}$ \\
\hline$T K$ & $\mathrm{~K}$ & 温度 Temperature & \\
\hline$T C$ & ${ }^{\circ} \mathrm{C}$ & 温度 Temperature & \\
\hline$R$ & $\mathrm{~J} \cdot \mathrm{K}^{-1} \cdot \mathrm{mol}^{-1}$ & 气体常数 Gas constant & 8.314 \\
\hline$S$ & $\mathrm{~J} \cdot \mathrm{K}^{-1} \cdot \mathrm{mol}^{-1}$ & 电子传输速率的温度参数 Entropy term & 650 \\
\hline$H$ & $\mathrm{~J} \cdot \mathrm{mol}^{-1}$ & 曲率参数 Deactivation energy & 200000 \\
\hline$C_{\mathrm{m}}$ & ${ }^{\circ} \mathrm{C}^{-1}$ & 温度修正参数 Temperature modification constant & \\
\hline$T_{\mathrm{m}}$ & ${ }^{\circ} \mathrm{C}$ & 温度修正参数 Temperature modification constant & \\
\hline$V_{\mathrm{Tm}}$ & $\mu \mathrm{mol} \cdot \mathrm{m}^{-2} \cdot \mathrm{s}^{-1}$ & 羧化速率参数 Rubisco carboxylase rate & \\
\hline$J_{\mathrm{Tm}}$ & $\mu \mathrm{mol} \cdot \mathrm{m}^{-2} \cdot \mathrm{s}^{-1}$ & 电子传输速率参数 Potential rate of electron transport & \\
\hline$N E E$ & $\mu \mathrm{mol} \cdot \mathrm{m}^{-2} \cdot \mathrm{s}^{-1}$ & 有效净生态系统 $\mathrm{CO}_{2}$ 交换率 Net ecosystem exchange & \\
\hline$P P F D$ & $\mu \mathrm{mol} \cdot \mathrm{m}^{-2} \cdot \mathrm{s}^{-1}$ & 有效光量子流密度 Photosynthetic photon flux density & \\
\hline$P_{2}$ & $\mathrm{kPa}$ & 大气压 Air pressure & \\
\hline$L A I$ & & 叶面积指数 Leaf area index & \\
\hline
\end{tabular}

可以接受的。需要注意的是, 因为我们用了不同的 温度修正方程, 得到的表1中方程(11)的 $V_{\mathrm{opt}}$ 和 $J_{\mathrm{opt}}$ 的 意义与表1中方程(10)的 $V_{25}$ 和 $J_{25}$ 的意义是不一样
的。仿效Medlyn等(2002b), 我们把方程(11)叫做速 率峰值方程。

通过图2, 可以初步看出模拟的效果。图中数据 


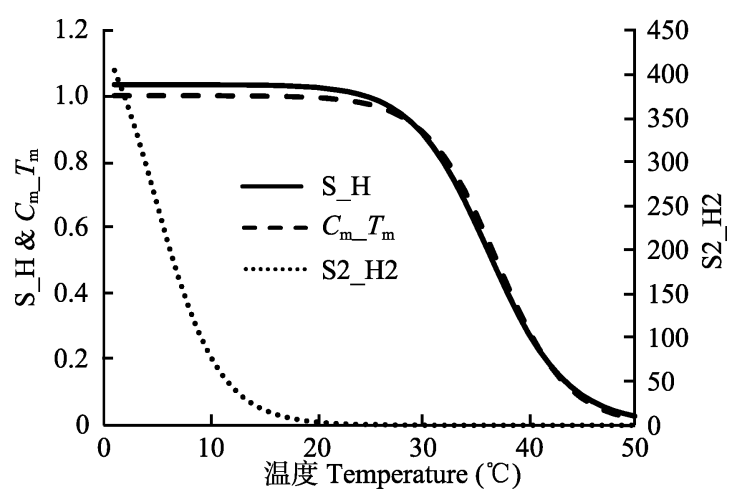

图1 温度修正模型比较。实线: von Caemmerer等(2009)的 曲线 $(S=710, H=220000)$, 点线: 增加 $S$ 约 $10 \%$ 的曲线 $(S=$ $790, H=220000)$, 虚线: 本文用的曲线 $\left(C_{\mathrm{m}}=0.3, T_{\mathrm{m}}=37\right)$ 。

Fig. 1 Comparison of the temperature correction models. Solid line is the curve of von Caemmerer et al. (2009) when $S$ $=710$ and $H=220000$; dotted line is the curve when $S$ is increased by $10 \%(S=790, \mathrm{H}=220000)$; dashed line is the curve calculated according to our new response curve $\left(C_{\mathrm{m}}=\right.$ $\left.0.3, T_{\mathrm{m}}=37\right)$.

表3 模型的参数的初始值范围和优化得到的值

Table 3 Initial parameter ranges and modeled values

\begin{tabular}{lllll}
\hline $\begin{array}{l}\text { 参数 } \\
\text { Parameter }\end{array}$ & $\begin{array}{l}\text { 参数值下限 } \\
\text { Lower value }\end{array}$ & $\begin{array}{l}\text { 参数值上限 } \\
\text { Upper value }\end{array}$ & $\begin{array}{l}\text { 实际模拟值 } \\
\text { Inversed value }\end{array}$ \\
\hline$V_{\mathrm{cmax}}$ & $V_{\mathrm{opt}}$ & 10 & 500 & 374 \\
& $E_{\mathrm{a}}$ & 10000 & 70000 & 21287 \\
$J_{\mathrm{max}}$ & $J_{\mathrm{opt}}$ & 10 & 500 & 310 \\
& $E_{\mathrm{a}}$ & 10000 & 50000 & 10000 \\
$R_{\mathrm{d}}$ & $R_{\mathrm{d} 25}$ & 0 & 10 & 10 \\
& $E_{\mathrm{a}}$ & 10000 & 70000 & 10033 \\
$C_{\mathrm{m}}$ & & 0.25 & 0.50 & 0.28 \\
$T_{\mathrm{m}}$ & & 20.0 & 50.0 & 26.9 \\
$g_{1}$ & & 0.0 & 10.0 & 3.7 \\
\hline
\end{tabular}

缩写同表2

Abbreviations see Table 2.

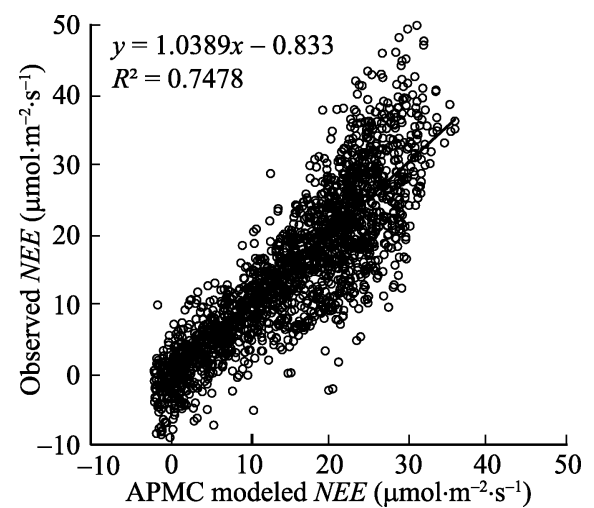

图2 净生态系统 $\mathrm{CO}_{2}$ 交换 $(N E E)$ 模拟结果(实线)与观测值 (符号)的比较。图中实线为 $1: 1$ 线。

Fig. 2 Relationship between modeled and observed net ecosystem exchange $(N E E)$. The solid line indicates that model outputs equal observations.

点一共1 948个, 考虑到浴度相关法测定时本身带有 一定的随机误差, 决定系数达到 0.75 体现了很好的
模拟效果。从斜率来看, 1.04 与 1 非常接近。以自由 度为 1946 的 $t$ 检验衡量, 达到极显著。 $N E E$ 的模拟与 观测平均值分别为15.66和15.32 $\mu \mathrm{mol} \cdot \mathrm{m}^{-2} \cdot \mathrm{s}^{-1}$, 标准 方差值分别为 9.08 和 $11.12 \mu \mathrm{mol} \cdot \mathrm{m}^{-2} \cdot \mathrm{s}^{-1}$, 二者之差 的平均值和标准方差分别为 0.34 和 $5.63 \mu \mathrm{mol} \cdot \mathrm{m}^{-2} \cdot \mathrm{s}^{-1}$ 。 这表明最佳粒子的参数给出了无偏差的 $N E E$ 模拟。

\section{3 讨论和结论}

虽然APMC方法在很多学科领域已有应用, 但 是在植物生态学上的应用比较少见。本文将重点放 在了介绍用APMC反演光合参数的技术细节, 而非 具体的植物生态学问题。笔者希望通过应用实例, 打开APMC方法在植物生理生态学研究中应用的大 门, 使该方法得到更广泛的应用。

利用APMC方法进行冠层光合参数反演的研究 还未见报道。过去光合参数的获得(这里指 $V_{\mathrm{cmax}}$ 和 $J_{\max }$ ), 大多是通过测定光合速率对胞间 $\mathrm{CO}_{2}$ 浓度上 升的响应曲线, 再结合Farquhar模型计算得到(详见 $\mathrm{Gu}$ 等(2010)及其参考文献)。正因如此, 笔者无法通 过对比本研究与其他方法所得的冠层参数值来说明 本方法的可靠性, 但是, 从模型在验证站点运行的 情况来看, 还是非常理想的。本文研究结果表明, 实 测值和根据反演参数模拟出来的数值具有很好的相 关性, 并且斜率接近 1 。考虑到浴度相关法本身就具 有较大的随机误差,该方法在具体应用上具有可行 性。实际上, 如果设定的参数初始值范围适当, $A P M C$ 方法几乎总是可以得出与观测 $N E E$ 有良好线 性关系的结果，做出非常好的模拟。我们同时还将 该方法应用在了叶片尺度以及其他通量站点, 也得 到了很好的拟合效果(未发表数据)。

但是, 使用APMC方法进行冠层光合参数反演, 并非完美。比如, 如果对参数初始值的随机取样范 围不加限制, 得到的参数值可能超出常识范围。这 不是参数估计方法的问题, 而是因为模型方程的过 度参数化(Medlyn et al., 2002b)。实际上, 因为植物 的光合速率取决于 $A_{\mathrm{c}}$ 和 $A_{\mathrm{j}}$ 中小的一个, 直接影响最 佳光合温度的参数就有 10 个。为了使曲线在拐点处 有很好的表现, 所选择的驱动变量,要有一定的梯度, 当梯度不足(比如高温高湿的热带雨林)时, 参数初 始值的选择就显得尤为重要。

本文将一种新的方法应用到已经成熟的光合作 用模型中, 完成参数反演的目的。从植物生态学的

www.plant-ecology.com 
角度看, 这个参数反演工作的重要性可以概括为以下 3点:

(1) 该方法有望成为叶片生理参数反演的一种 新选择。长期以来, $V_{\mathrm{cmax}}$ 和 $J_{\max }$ 都是通过测定 $\mathrm{CO}_{2}$ 浓 度响应曲线, 结合Farquhar模型计算出来的。进行 $\mathrm{CO}_{2}$ 浓度响应曲线的测定, 操作比较复杂, 并且基 于这些数据来计算参数有较大的不确定性(Gu et al., 2010)。而我们的方法可以通过日常条件下的一系列 监测值来实现反推生理参数的目的, 可以作为叶片 生理参数反演的一种新选择。

\section{(2) 使直接获得冠层整体生理参数成为可能。} 对森林植被而言, 想要对整个冠层进行控制, 进行 $\mathrm{CO}_{2}$ 响应分析, 在目前的技术条件下几乎没有可行 性。然而, 我们这个方法提供了这种可能性, 利用浴 度相关技术所测定的生态系统净 $\mathrm{CO}_{2}$ 交换量即可反 推出整个冠层的光合生理参数。这是一个全新的视 角, 对深入挖掘和利用浴度通量数据, 以及获得冠 层生理参数都有重要价值。

(3) 有益于叶片到冠层尺度扩展问题的解决。 如何将叶片尺度的机理模型应用到由众多树种组成 的森林冠层上, 一直都没有很好的解决办法。一个 比较传统的方法是通过选择典型树种向阳的几片代 表性的叶片, 用光合叶室测定 $\mathrm{CO}_{2}$ 浓度响应曲线来 推导出这几个种的光合生理参数值, 然后将平均值 代入叶片尺度模型, 进行计算模拟。不论是多层模 型还是大叶模型, 基本上都采用这种思路。对于农 田生态系统而言, 物种单一, 冠层低矮, 在实际的 应用中, 上述方法尚有一定的可行性。对于森林冠 层, 特别是物种多样的热带森林, 该方法的应用则 存在诸多困难。一方面, 要进入高达 $80 \mathrm{~m}$ 的林冠层 进行 $\mathrm{CO}_{2}$ 浓度响应曲线的测量是非常困难的; 另一 方面, 热带森林物种多样, 很难确定到底选哪些种、 取多少种能代表这个生态系统。本研究所提出的方 法, 为这个问题的解决提供了一个新的选择。 APMC模型结合方法机理明确, 模拟效果好。这个 方法的提出和应用对光合模型参数的获取或许有积 极的意义, 为解决长期困扰我们的尺度扩展问题提 供了新的视角。

\section{基金项目 国家自然科学基金(31200347和 31660142)。}

致谢 感谢日本的苫小牧观测站提供观测数据。

\section{参考文献}

Amthor JS (1994). Scaling $\mathrm{CO}_{2}$-photosynthesis relationships from the leaf to the canopy. Photosynthesis Research, 39, 321-350.

Andrieu C, Doucet A, Holenstein R (2010). Particle Markov chain Monte Carlo methods. Journal of the Royal Statistical Society, Series B, 72, 269-342.

Baldocchi D (1994). An analytical solution for coupled leaf photosynthesis and stomata1 conductance models. Tree Physiology, 14, 1069-1079.

Ball JT, Woodrow JT, Berry JA (1987). A model predicting stomatal conductance and its contribution to the control of photosynthesis under different environmental conditions. In: Biggins J ed. Progress in Photosynthesis Research, Vol. 4. Proceedings of the 7th International Congress on Photosynthesis. Matins Nijhoff, Dordrecht, the Netherlands. 221-224.

Beaumont MA (2010). Approximate Bayesian computation in evolution and ecology. Annual Review of Ecology, Evolution, and Systematics, 41, 379-406.

Csilléry K, Blum MGB, Gaggiotti OE, Francois O (2010). Approximate Bayesian Computation (ABC) in practice. Trends in Ecology and Evolution, 25, 410-418.

Cubasch U, Wuebbles D, Chen D, Facchini MC, Frame D, Mahowald N, Winther JG (2013). Introduction. In: Stocker TF, Qin D, Plattner GK, Tignor M, Allen SK, Boschung J, Nauels A, Xia Y, Bex V, Midgley PM eds. Climate Change 2013: The Physical Science Basis. Cambridge University Press, Cambridge, UK.

Dai Y, Dickinson RE, Wang YP (2004). A Two-Big-Leaf Model for canopy temperature, photosynthesis, and stomatal conductance. Journal of Climate, 17, 2281-2299.

Damour G, Simonneau T, Cochard H, Urban L (2010). An overview of models of stomatal conductance at the leaf level. Plant, Cell \& Environment, 33, 1419-1438.

de Pury DGG, Farquhar GD (1997). Simple scaling of photosynthesis from leaves to canopies without the errors of big-leaf models. Plant, Cell \& Environment, 20, 537-557.

Farquhar GD, von Caemmerer S, Berry JA (1980). A biochemical model of photosynthetic $\mathrm{CO}_{2}$ assimilation in leaves of $\mathrm{C}_{3}$ species. Planta, 149, 78-90.

Gao M, Zhang H (2012). Sequential Monte Carlo methods for parameter estimation in nonlinear state-space models. Computers and Geosciences, 44, 70-77.

Groenendijk M, Dolman AJ, Ammann C, Arneth A, Cescatti A, Dragoni D, Gash JHC, Gianelle D, Gioli B, Kiely G, Knohl A, Law BE, Lund M, Marcolla B, van der Molen MK, Montagnani L, Moors E, Richardson AD, Roupsard O, Verbeeck H, Wohlfahrt G (2011). Seasonal variation of photosynthetic model parameters and leaf area index from global Fluxnet eddy covariance data. Journal of Geophysical doi: 10.17521/cjpe.2016.0067 
Research, 116, G04027. doi:10.1029/2011JG001742.

Gu L (2010). Reliable estimation of biochemical parameters from $\mathrm{C}_{3}$ leaf photosynthesis-intercellular carbon dioxide response curves. Plant, Cell \& Environment, 33, 18521874.

Hartig F, Dislich C, Wiegand T, Huth A (2014). Technical note: Approximate Bayesian parameterization of a process-based tropical forest model. Biogeosciences, 11, 1261-1272.

Hirano T, Hirata R, Fujinuma Y, Saigusa N, Yamamoto S, Harazono Y, Takada M, Inukai K, Inoue G (2003). $\mathrm{CO}_{2}$ and water vapor exchange of a larch forest in northern Japan. Tellus, 55B, 244-257.

Jarvis PG (1995). Scaling processes and problems. Plant, Cell \& Environment, 18, 1079-1089.

June T, Evans JR, Farquhar GD (2004). A simple new equation for the reversible temperature dependence of photosynthetic electron transport: A study on soybean leaf. Functional Plant Biology, 31, 275-283.

Kosugi Y, Takanashi S, Ueyama M, Ohkubo S, Tanaka H, Matsumoto K, Yoshifuji N, Ataka M, Sakabe A (2013). Determination of the gas exchange phenology in an evergreen coniferous forest from 7 years of eddy covariance flux data using an extended big-leaf analysis. Ecological Research, 28, 373-385.

Lenormand M, Jabot F, Deffuant G (2013). Adaptive approximate Bayesian computation for complex models. Computational Statistics, 28, 2777-2796.

MacEachern SN, Clyde M, Liu J (1999). Sequential importance sampling for nonparametric Bayes models: The next generation. Canadian Journal of Statistics, 27, 251-267.

Marin JM, Pudlo P, Robert CP, Ryder RJ (2012). Approximate Bayesian computational methods. Statistics and Computing, 22, 1167-1180.

Medlyn BE, Dreyer E, Ellsworth D, Forstreuter M, Harley PC, Kirschbaum MUF, Le Roux X, Montpied P, Strassemeyer J, Walcroft A, Wang K, Loustau D (2002a). Temperature response of parameters of a biochemically based model of photosynthesis. II. A review of experimental data. Plant, Cell \& Environment, 25, 1167-1179.

Medlyn BE, Loustau D, Delzon S (2002b). Temperature response of parameters of a biochemically based model of photosynthesis. I. Seasonal changes in mature maritime pine (Pinus pinaster Ait.). Plant, Cell \& Environment, 25, 1155-1165.
Saigusa N, Yamamoto S, Hirata R, Ohtani Y, Ide R, Asanuma J, Gamo M, Hirano T, Kondo H, Kosugi Y, Li S-G, Nakai Y, Takagi K, Tani M, Wang H (2008). Temporal and spatial variations in the seasonal patterns of $\mathrm{CO}_{2}$ flux in boreal, temperate, and tropical forests in East Asia. Agricultural and Forest Meteorology, 148, 700-713.

Sellers PJ, Randall DA, Collatz GJ, Berry JA, Field CB, Dazlich DA, Zhang C, Collelo GD, Bounoua L (1996). A revised land surface parameterization (SiB2) for atmospheric GCMs. Part 1: Model formulation. Journal of Climate, 9, 676-705.

Sprintsin M, Chen JM, Desai A, Gough CM (2012). Evaluation of leaf-to-canopy upscaling methodologies against carbon flux data in North America. Journal of Geophysical Research, 117, G01023. doi:10.1029/2010JG001407.

Toni T, Welch D, Strelkowa N, Ipsen A, Stumpf MPH (2009). Approximate Bayesian computation scheme for parameter inference and model selection in dynamical systems. Journal of the Royal Society Interface, 6, 187-202.

von Caemmerer S, Farquhar G, Berry J (2009). Biochemical model of $\mathrm{C}_{3}$ photosynthesis. In: Laisk A, Nedbal L, Govindjee eds. Photosynthesis in Silico: Understanding Complexity from Molecules to Ecosystems. Springer, Dordrecht, the Netherlands. 209-230.

Vrugt JA, Sadegh M (2013). Toward diagnostic model calibration and evaluation: Approximate Bayesian computation. Water Resources Research, 49, 4335-4345.

Wang H, Saigusa N, Yamamoto S, Kondo H, Hirano T, Toriyama A, Fujinuma F (2004). Net ecosystem $\mathrm{CO}_{2}$ exchange over a larch forest in Hokkaido, Japan. Atmospheric Environment, 38, 7021-7032.

Wang YP, Leuning R (1998). A two-leaf model for canopy conductance, photosynthesis and partitioning of available energy I: Model description and comparison with a multilayered model. Agricultural and Forest Meteorology, 91, 89-111.

Xu L, Baldocchi DD (2003). Seasonal trends in photosynthetic parameters and stomatal conductance of blue oak (Quercus douglasii) under prolonged summer drought and high temperature. Tree Physiology, 23, 865-877.

Yin X, Struik PC (2009). $C_{3}$ and $C_{4}$ photosynthesis models: An overview from the perspective of crop modelling. Wageningen Journal of Life Sciences, 57, 27-38.

责任编委: 张金屯 责任编辑: 李 敏

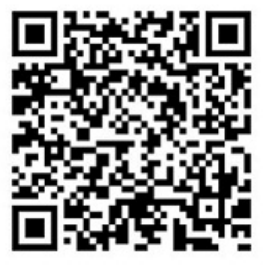

扫码向作者提问

www.plant-ecology.com 
附录 I APMC 在本文中的具体实现

Appendix I Model entity in the APMC

对每一个 APMC 粒子 For all particles

for $k=1$ to $\mathrm{N}_{\mathrm{obs}}$ (对所有的观测数据) For all observations

用 APMC 粒子选定的 $V_{25}, J_{25}, R_{\mathrm{d} 25}, g_{1}, E_{\mathrm{a}}, C_{\mathrm{m}}, T_{\mathrm{m}} \quad$ Use parameter values selected by AMPC

计算 $V_{\mathrm{cmax}}, J_{\max }, R_{\mathrm{d}}, g_{\mathrm{b}} \quad$ Estimate target model parameters

设 $t=1\left(C_{\mathrm{i}}\right.$ 的迭代计算次数 $)$ At initial time

设 $C_{\mathrm{i}}^{0}=0.7 C_{\mathrm{a}} \quad$ Set the intercellular $\mathrm{CO}_{2}$ equal to $70 \%$ of air $\mathrm{CO}_{2}$

设 $\Delta C_{\mathrm{i}}>1$ Set the intercellular $\mathrm{CO}_{2}$ not in equilibrant

while $\Delta C_{\mathrm{i}}>1$ do

计算 $A_{\mathrm{c}}, A_{\mathrm{j}}$ Compute the two rate.

计算 $A=\frac{A_{\mathrm{c}}+A_{\mathrm{j}}-\sqrt{\left(A_{\mathrm{c}}+A_{\mathrm{j}}\right)^{2}-4 \times 0.98 A_{\mathrm{c}} A_{\mathrm{j}}}}{2 \times 0.98} \quad$ Compute the joint rate

计算 $C_{\mathrm{i}}^{t}=C_{\mathrm{a}}-\frac{A}{g_{\mathrm{b}}}-\frac{g_{\mathrm{b}} C_{\mathrm{a}} A-A^{2}}{g_{\mathrm{b}} g_{1} h_{s} A-g_{0} A+g_{\mathrm{b}} g_{0} C_{\mathrm{a}}} \quad$ Compute the intercellular $\mathrm{CO}_{2}$

$\Delta C_{\mathrm{i}}=\left|C_{\mathrm{i}}^{t}-C_{\mathrm{i}}^{t-1}\right| \quad$ Check equilibrant state

设 $t=t+1 \quad$ Advance time

end while

\section{end for}

将 $|A-N E E|$ 的平均值作为 APMC 粒子的 $\rho$ Calculate the difference between modeled photosynthesis rate and the observed rate 